\section{and Functional}

Neurosurgery
Stereotact Funct Neurosurg 2021;99:150-158

DOI: 10.1159/000511202
Received: May 12, 2020

Accepted: August 24, 2020

Published online: September 30, 2020

\title{
Longitudinal Assessment of Rotation Angles after Implantation of Directional Deep Brain Stimulation Leads
}

\author{
Florian Lange $^{\mathrm{a}}$ Frank Steigerwald $^{\mathrm{a}}$ Doortje Engel $^{\mathrm{b}}$ Tobias Malzacher $^{\mathrm{a}}$ \\ Tilmann Neun ${ }^{b}$ Patrick Fricke ${ }^{c}$ Jens Volkmann ${ }^{a}$ Cordula Matthies ${ }^{c}$ \\ Philipp Capetian ${ }^{a}$
}

${ }^{a}$ Department of Neurology, University Hospital Würzburg, Würzburg, Germany; ${ }^{b}$ Department of Neuroradiology, University Hospital Würzburg, Würzburg, Germany; ${ }^{c}$ Department of Neurosurgery, University Hospital Würzburg, Würzburg, Germany

\section{Keywords}

Deep brain stimulation · Directional leads · Rotation angles · Cranial computer tomographies · Rotation fluoroscopy

\begin{abstract}
Purpose: The risk/benefit-ratio of deep brain stimulation (DBS) depends on focusing the electrical field onto the target volume, excluding side-effect eliciting structures. Directional leads limiting radial current diffusion can target stimulation but add a spatial degree of freedom that requires control to align multimodal imaging datasets and for anatomical interpretation of stimulation. Unpredictable postoperative lead rotations have been reported. The extent and timing of rotation from the surgically intended alignment remain uncertain, as does the time point at which directional stimulation can be safely initiated without risking unexpected shifts in stimulation volume. We present a retrospective analysis of clinically indicated, repeated neuroimaging controls postimplantation in patients with directional DBS systems, which allow estimation of the amount and timing of postoperative lead rotation. Methods: Data from 67 patients with directional leads and multiple cranial computer tomographies (CCT) and/or rotation fluoroscopies at different postopera-
\end{abstract}

karger@karger.com www.karger.com/sfn

Karger $\frac{1}{6}$

GOPEN ACCESS
(C) 2020 The Author(s)

Published by S. Karger AG, Basel

This is an Open Access article licensed under the Creative Commons Attribution-NonCommercial-4.0 International License (CC BY-NC) (http://www.karger.com/Services/OpenAccessLicense), applicable to the online version of the article only. Usage and distribution for commercial purposes requires written permission. tive time points were included. Rotation angles were detected based on CCT artifacts ( $n=56$ ) or direct visualization of lead segments on rotation fluoroscopies $(n=52)$. Cross-validation of both methods was conducted in patients who received both imaging modalities $(n=51)$. Results: Rotation angles deviated significantly $\left(\sim 30^{\circ}\right)$ from their intended $0^{\circ}$ anterior/posterior orientation. Rotation was firmly established within the first postoperative day, with no additional torque in subsequent scans. The two methods highly correlated (right hemisphere: $R^{2}=0.94$, left hemisphere: $R^{2}=0.91$ ). Conclusion: Both methods for measuring rotation angles led to comparable results and can be used interchangeably. Directional stimulation settings can safely be initiated after the first postoperative day, without risking subsequent lead rotation-related anatomical shifts. @ 2020 The Author(s)

Published by S. Karger AG, Basel

\section{Introduction}

Deep brain stimulation (DBS) as a therapeutic approach to movement disorders such as Parkinson disease (PD), dystonia, and tremor has always relied on neuroimaging for precise lead placement [1]. Still, to date, no gen- 
erally accepted protocol for lead localization has been established, and most clinicians use imaging fusion of preand postoperative cranial computer tomography (CCT) and/or magnetic resonance imaging (MRI) data to confirm the lead location at the attempted site [2]. However, for many years programming DBS has largely relied on clinical testing of the individual contacts and finding thresholds for beneficial effects and adverse effects (the so-called "monopolar review session") [3, 4]. Only recently have image-guided programming approaches attracted increasing attention based on computational models of current diffusion within a patient-specific anatomical model of the lead location and target structures [5].

The recent introduction of directional leads provides the opportunity of steering the electrical field in a horizontal mode, and it widens the options for individual shaping of the volume of tissue activated in vertical directions. It adds opportunities for spatially restricted current diffusion but also for complexities in programming. The more focused delivery of the stimulation current potentially widens the therapeutic window by decreasing efficacy thresholds while increasing side effect thresholds [6]. With conventional, ring-shaped leads, which emit the current in a spherical shape, the rotation angle of the leads does not matter for the programmer. However, with segmented leads precise alignment between lead geometry and the brain coordinate system is of the utmost importance, as it determines the contacts and spatial direction in which the current is being emitted. Segmentation of circular into directional contacts increases the number of programmable contacts and complicates the clinical review and programming process. Simulation of the electrical stimulation field in relation to surrounding anatomical structures, visualized by individual neuroimaging with the help of specialized computer software, is the basis for "image-guided programming." It can potentially lead to more tailored stimulation programs and save time for patients and programmers. Image-guided programming with directional leads requires knowledge about the lead trajectory, implant depth, and lead orientation. While the first 2 parameters are determined by the stereotactic insertion technique, lead orientation remains uncontrolled and needs to be manually established during the operation by aligning an external lead marker with the $y$-axis of the stereotactic frame facing anteriorly. However, the leads are not rigid and may allow some torsion between the external marker and the lead level [7]. Moreover, as documented in a recent study by Dembek et al. [8], un- predictable postoperative lead rotations may occur after bending the lead for fixation (giving rise to internal spring forces from the wires running in a clockwise rotation within the leads) and because of lead movements due to brain pulsation.

It is currently unclear when this lead rotation takes place and when the final position is reached. Thus, there is uncertainty regarding the time point after the operation when initiation of directional programming (clinical or imaging-based) would be sensible and when directional settings would need to be reviewed and possibly readjusted during follow-up. Therefore, we decided to retrospectively look at the routine postoperative CCT and repeated imaging due to medical indications in some of our patients to allow us to gain insight in the timing and extent of lead rotation during follow-up.

\section{Materials and Methods}

\section{Data Collection}

We retrospectively analyzed all of the patients in our center with implanted Cartesia ${ }^{\circledR}$ directional DBS leads (Boston Scientific, Marlborough, MA, USA) between September 2015 and June 2019 $(n=123)$. We included all patients with at least 2 postoperative cranial imaging datasets (CCT and/or rotation fluoroscopies) ( $n=$ $65 ; 126$ leads; note that this did not include intraoperative monoplanar fluoroscopy). The clinical indications for neuroimaging were postoperative controls or verification of lead position, determination of the rotation angles for the initial programming, or, in the case of serial images, due to unexpected stimulation-induced side effects or waning therapeutic effects.

\section{Surgical Placement of Leads}

All operations were performed by 2 neurosurgeons (C.M. and P.F.). Consistency was ensured by following a standardized clinical protocol. Landmark-based preoperative planning was carried out based on nonstereotactic MRI acquired under deep sedation using Brainlab Elements (Surgiplan 3.0; Elekta, Stockholm, Sweden, and/or Brainlab AG, Munich, Germany). The operation procedure started with the placement of a Leksell ${ }^{\circledR}$ stereotactic frame (Elekta, Stockholm, Sweden) under general anesthesia. Then patients underwent a cranial CT scan, which was digitally fused with the preoperative MRI (Surgiplan 3.0, Elekta, and/or Brainlab Elements, Brainlab AG). The hemisphere corresponding to the more severely affected body side in $\mathrm{PD}$, focal dystonias, and lateralized tremor would be operated on first. In the case of a very symmetric symptom manifestation, the dominant hemisphere would be the first to be operated. Stereotactic lead placement was guided by multitrajectory microrecordings, test stimulation, and clinical assessment. The directional lead (Cartesia ${ }^{\circledR}$; Boston Scientific) was inserted into the neurophysiologically determined path. The intended anterior side of the lead was identified beforehand and marked by a lead stopper so that the lead could be inserted facing forward. If not facing anteriorly after insertion, the radiopaque marker was oriented anteriorly under lateral fluoroscopy. The lead was fixed 


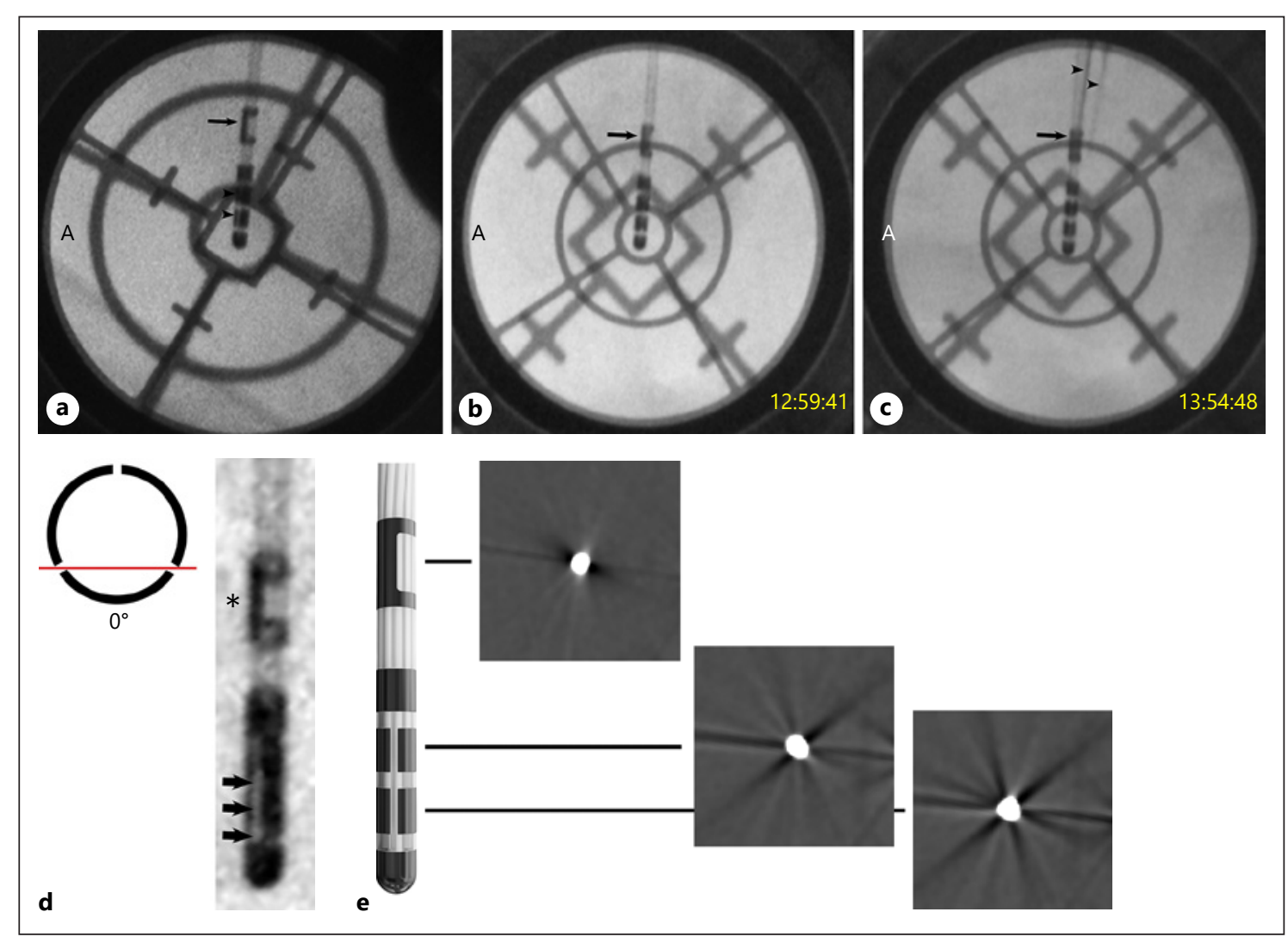

Fig. 1. Overview of the employed imaging modalities. a Lateral fluoroscopy with a C-ring was used for intraoperative visualization of the lead and its directional marker (arrow). If the optimal anterior placement was achieved ( $\mathrm{A}=$ anterior), the spaces between the directional contacts of the 2 middle contacts appeared as thin lines (arrowheads). b Image taken after definitive fixation of the first directional lead: the directional marker (arrow) indicates a forward-facing lead (yellow numbers = time image was taken). $\mathbf{c}$ Next image taken approximately $1 \mathrm{~h}$ after insertion of the microleads for recordings (arrowheads): the directional marker (arrow) clearly

at the burr hole level with forceps until it was fixated to the skull with titanium plates and screws. During the individual steps of lead placement, lateral radiographs were taken and saved for documentation (Fig. 1a-c). Finally, lead extensions and the stimulator were implanted under general anesthesia.

\section{Image Acquisition}

All of the patients received their initial postoperative CCT imaging within $24 \mathrm{~h}$ after surgery as part of the routine clinical protocol to exclude peri- or postoperative complications. From May 2018, an additional rotation fluoroscopy [7] has routinely been used at our institution to determine the lead orientation. In March 2019, CCT artifact-based identification of the rotation angles [9] was established, and it was also employed to retrospectively determine the rotation angles for this study. Some patients received additional imaging if clinically required. indicates lead rotation from the position in the last image. $\mathbf{d}$ The spaces between the directional contacts allow unobstructed passage of the X-ray beam (red line). Like aiming through iron sights, a thin line (arrows) results in the rotation fluoroscopy every $30^{\circ}$. Together with the $\mathrm{c}$-shaped directional marker (asterisk), the exact lateral angulation of the X-ray beam to the lead can be identified. e The DiODe algorithm employs CCT artifacts at the levels of the directional marker (upper image) and the 2 directional contacts (lower pictures) to predict the lead rotation (image of the directional lead provided courtesy of Boston Scientific).

Conventional CCT was performed with a Somatom Definition AS (Siemens, Erlangen, Germany) with a slice thickness of $0.8 \mathrm{~mm}$. Rotation fluoroscopy was performed with an Artis Q system (Siemens) from $-120^{\circ}$ to $+120^{\circ}$ with a spatial resolution of $0.47 \mathrm{~mm}$.

\section{Determination of Lead Rotation}

The rotation of the directional leads was determined based on 1 or both of the 2 imaging modalities, i.e., postoperative CCT imaging or rotation fluoroscopy.

To determine the orientation of the directional DBS lead via rotation fluoroscopy, we used the "iron sight" method as described by Reinacher et al. [7], i.e., the small gaps between the directional segments can be identified in the fluoroscopy (iron sights). These gaps, which overlap every $60^{\circ}$, in conjunction with the directional marker of the leads were used to calculate the rotation of the DBS lead (Fig. 1d). In most cases, we employed the $\pm 90^{\circ}$ iron sight, which can be readily identified by the characteristic c-shaped ap- 
pearance of the directional marker at this position. In the case of rotation angles close to $0^{\circ}$, superimposed leads prohibited determination of the rotation angle with this iron sight. However, since iron sights appear every $60^{\circ}$, identifying either the one before or the one after and adding or subtracting $60^{\circ}$ solved this problem.

The projection of the internal acoustic meatus or the projection of the nasal crest and occipital protuberance was used to correct for deviations in head placement. We used the viewer module of the Brainlab Elements software to view and identify rotation angles (Brainlab Elements; Brainlab AG).

To determine the orientation via CCT, we used the DiODe algorithm developed by Hellerbach et al. [9]. DiODe as part of the Lead DBS software [10] uses the characteristic CT artifacts at 3 different levels of the DBS lead (the rotation marker and each level of segmented contacts) to identify the rotation angle (Fig. 1e).

\section{Statistics}

All statistical calculations were performed using GraphPad Prism 8 software (GraphPad Software, Inc., San Diego, USA). $p<$ 0.05 was considered statistically significant. The KolmogorovSmirnov test was employed for testing the normal distribution of values. When testing for a significant deviation of lead rotations from $0^{\circ}$, all values in their dimensionless form were calculated separately for the left and right sides using the one-sample Wilcoxon test. Testing for significant differences between leads in the right and left hemispheres was performed by a mixed-effects analysis with the multiple-comparisons test of Holm-Sidak. For visualization of the rotation angle distribution, percentages of $30^{\circ}$ ranges were calculated and the mean values of all leads by both methods were visualized using Simplex Numerica Software (Simplex Programs IT, Jülich, Germany). The patient group with 2 rotation fluoroscopies was divided into patients with the first of the 2 images acquired within the first postoperative week (F1 and F2) and patients with their first rotation fluoroscopy taken at a later stage (F3 and F4). Because of missing values due to patients with single leads, values for the left and right hemispheres were compared using a mixed-effects analysis with the multiple-comparisons test of Holm-Sidak. The Pearson correlation coefficient between rotation angles determined by CT and fluoroscopy was computed separately for each hemisphere. For PD patients with available clinical data, the lateralized motor outcome was determined by adding up scores of items $3-8$ and $15-17$ of the MDS-UPDRSIII. The percentage of improvement or deterioration rated by MedOFF/ StimON after 12 months versus MedOFF preoperative was calculated and correlated to the dimensionless value of rotation angles of the contralateral leads by the Spearman correlation coefficient. Data was only included in the analysis for a given lead if directional contacts were activated according to the session reports from the time when the clinical evaluation was performed.

Where appropriate, results are presented as means \pm SEM.

\section{Results}

\section{Patient Data}

In total, 65 patients with 126 directional leads were analyzed (see Fig. 2 for a detailed overview of the patient cohort, the received imaging, and the subdivision into

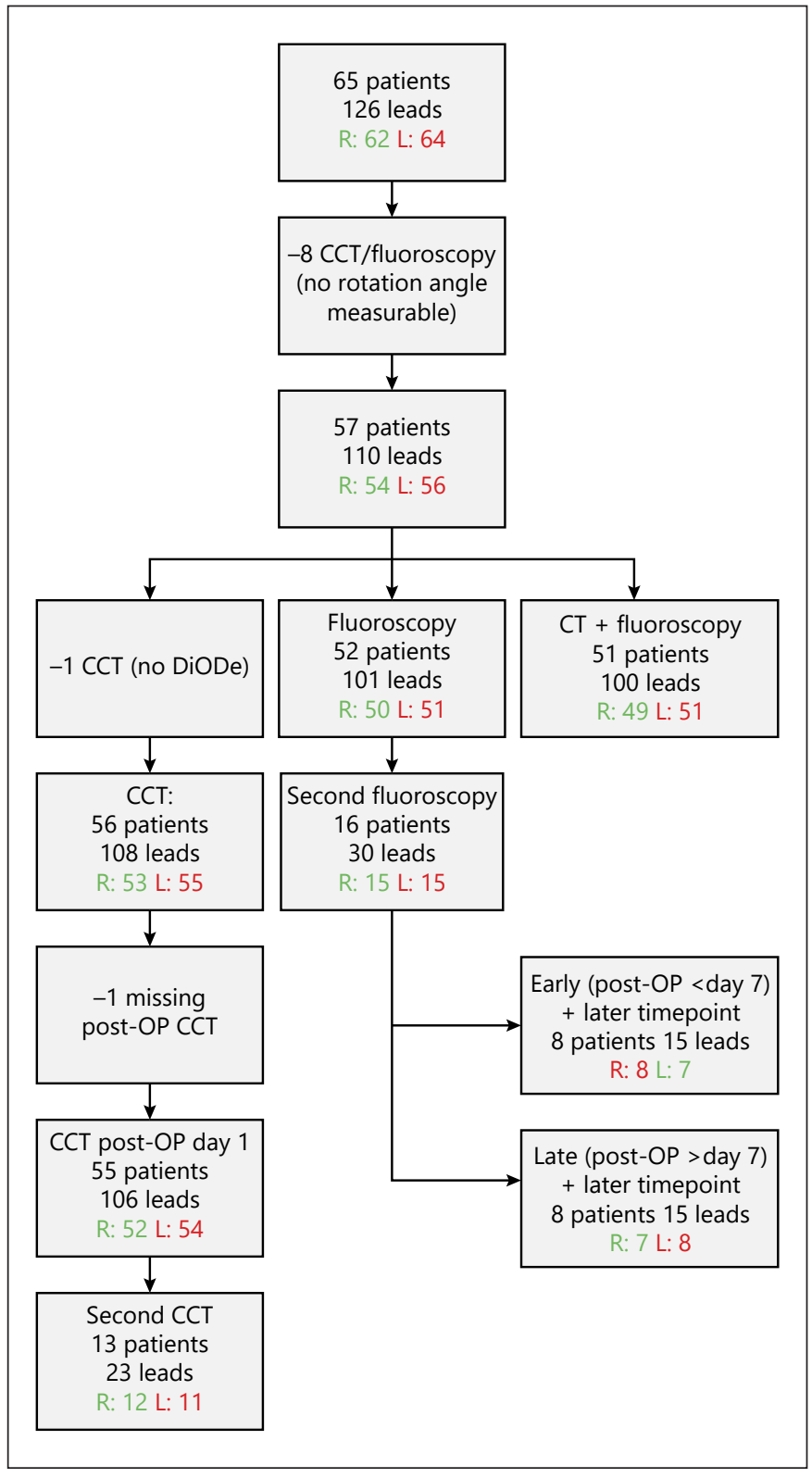

Fig. 2. Overview of the patient cohort and individual groups, with patient numbers per group. R, right; L, left.

statistical groups). Of these leads, 106 had been implanted in the subthalamic nucleus, 10 in the internal part of the globus pallidus, and 10 in the thalamic ventral intermediate nucleus/posterior subthalamic area. Three patients (2 who received CCT and rotation fluoroscopy and 1 who received only CCT) had only 1 lead in place, leading to an uneven distribution of the left and right hemispheric leads. Detailed information on the course of operation was available for 97 implanted leads and 107 impulse gen- 

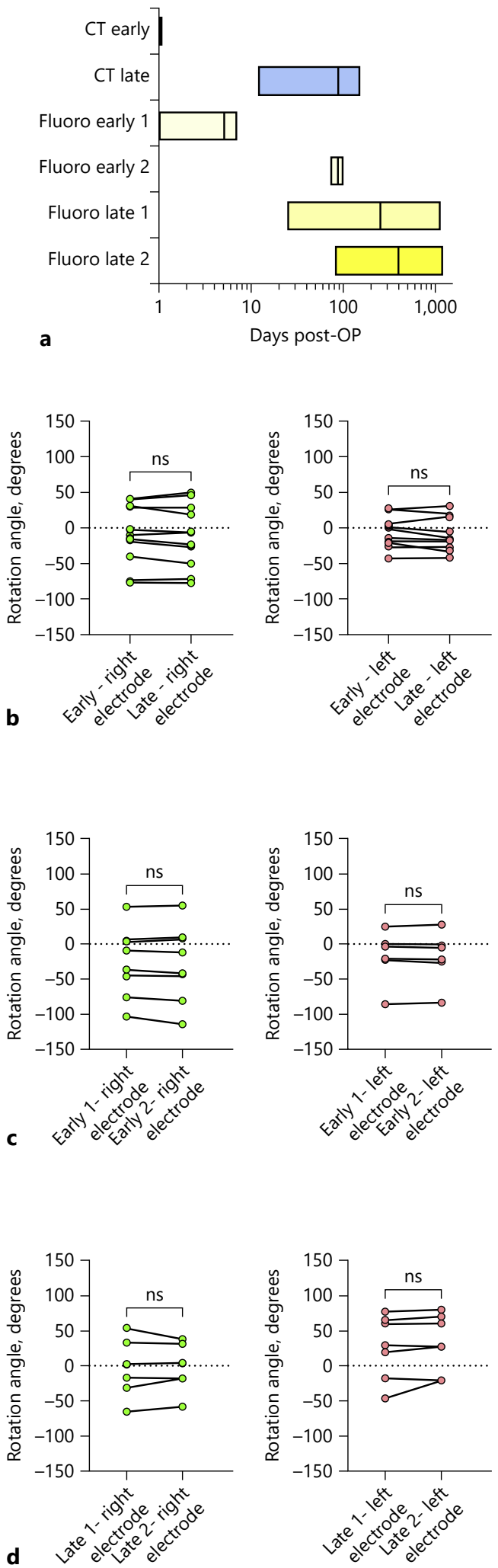

erator implantations in total. Of these, 31 leads $(32 \%)$ were implanted into the right hemisphere first and 66 (68\%) were implanted into the left hemisphere first. Sixty-three impulse generators were implanted into the right body side $(58.88 \%)$ and 44 were implanted into the left (41.12\%).

The imaging quality of the intraoperative lateral fluoroscopies was sufficient to identify the proximal directional marker (arrow in Fig. 1a) and, under optimal conditions, the iron sights (arrowheads in Fig. 1a). In 2 cases, comparison of the image immediately after fixation of the first lead (Fig. 1b) compared with the image taken at insertion of the microleads on the second side (arrowheads in Fig. 1c) revealed a substantial change in the appearance of the directional marker, indicating a significant rotation of the lead within less than $1 \mathrm{~h}$.

The rotation fluoroscopies of 8 patients $(12.3 \%)$ and the CCT of 9 patients (13.4\%) had to be excluded due to insufficient image quality for measuring rotation angles. All of the excluded images exhibited a lead angle that was too shallow when the images were taken, which resulted in insufficient definition of both the CT artifacts and the iron sights of the rotation fluoroscopies.

CCT images were available for 55 patients (106 leads) on the first postoperative day (CT early); 1 patient was not operated on in our institution and therefore no postoperative CCT was available). Thirteen patients (23 leads) received a second CCT 3 months later (CT late; $88 \pm 10$ days postoperatively).

Rotation fluoroscopy was available for 52 patients (101 leads, $352 \pm 50$ days postoperatively) and 16 patients (30 leads) received a second fluoroscopy on average 8 months after the first one ( $243 \pm 72$ days postoperatively).

For further analysis, we subdivided the patients with 2 rotation fluoroscopies into 2 groups with imaging at 4

Fig. 3. Rotation angles of individual leads at different time points. a Overview of the different imaging time points in the individual groups. b No significant changes in rotation angles as measured by the CCT method between the early time point (postoperative day 1 ) and later time points (right: $p=0.45$; left: $p=0.83$; mixed-effects analysis with the Holm-Sidak multiple-comparisons test). c As determined by rotation fluoroscopy, there are no significant differences in rotation angles between the first (early 1) and second time points (early 2) of rotation fluoroscopy imaging (right: $p=0.6$; left: $p=0.9$; mixed-effects analysis with the Holm-Sidak multiple comparison test). d Differences in rotation angles between the third ("late 1") and fourth group of imaging time points ("late 2") for rotation fluoroscopy are also non-significant (right: $p=0.98$; left: $p=0.21$; mixed-effects analysis with Holm-Sidak's multiple comparison test) 


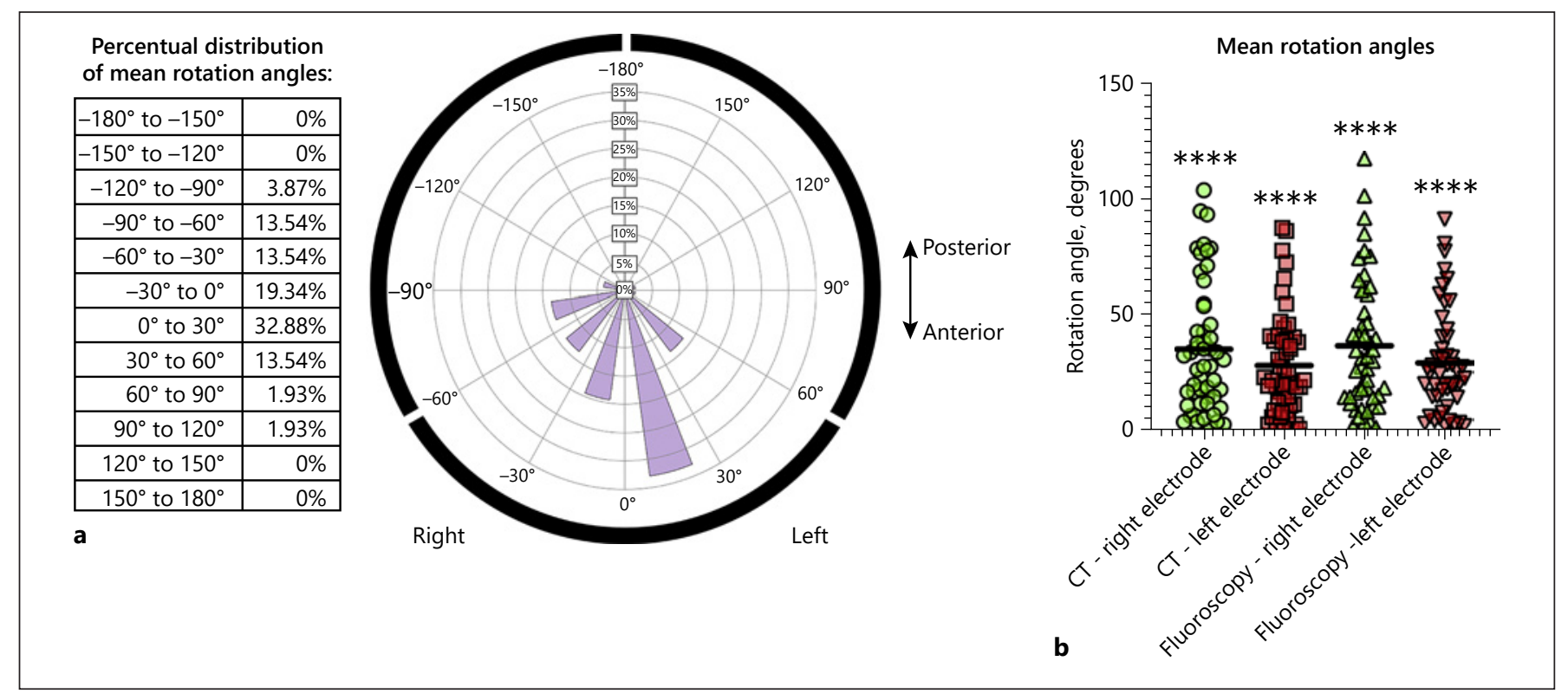

Fig. 4. Distribution of lead rotation angles. a The distribution of the mean rotation angles from both hemispheres and both modalities shows a majority of leads facing in the intended anterior direction, with considerable deviations in a number of leads. b Individual rotation angles (dimensionless) and mean values (thick line) separated by imaging modality and side. The mean values exhibited in all cases a highly significant deviation from the intended $0^{\circ}$ orientation $\left({ }^{* * * *} p<000.1\right.$ in all cases, Wilcoxon signed-rank test). No significant differences between left and right leads could be observed for either modalities ( $p=0.28$ for both modalities, mixed-effects with the Holm-Sidak multiple-comparisons test).

different time points (Fig. 3a): The first group received their first rotation fluoroscopy (fluoro early 1) during their postoperative hospital stay within the first week $(8$ patients, 15 leads, first imaging $5 \pm 0.68$ days postoperatively). In case of a relevant clinical deterioration or problematic side effects at their first postoperative visit in our department, a second rotation fluoroscopy was done (fluoro early 2) to rule out delayed lead rotation, which would change the relative orientation of the electric field to target structures $(87.75 \pm 3.79$ days postoperatively). The second group received their first rotation fluoroscopy (fluoro late 1) once we established this method in our institution ( $253.8 \pm 135.9$ days postoperatively) with the intention of optimizing programming parameters. The second fluoroscopy in this group (fluoro late 2) was made if clinical deterioration was observed or undesired side effects emerged (396.8 \pm 129.1 days postoperatively).

\section{Global Overview on Lead Rotation}

We observed postoperative lead rotations between $-103^{\circ}$ to $116.8^{\circ}$ from the intended $0^{\circ}$ using all imaging modalities (between $-106^{\circ}$ and $94^{\circ}$ for the rotation fluo- roscopy and between $-100^{\circ}$ and $116.8^{\circ}$ for the CCT) (Fig. 4a); almost exactly $50 \%$ of all of the leads rotated to either the left or the right (to the right: $\leq 0^{\circ}, 49.72 \%$; to the left: $\left.\geq 0^{\circ}, 50.28 \%\right)$. Rotation angles were transformed into dimensionless values for further analysis, since otherwise the clockwise and counterclockwise rotations would average out to $0^{\circ}$; the mean rotation angle of all leads was roughly $30^{\circ}$ (Fig. 4 b; CCT: right, $34 \pm 3.9^{\circ}$ [ $n=52$ ]; left, $27 \pm 3^{\circ}[n=54]$; rotation fluoroscopy: right, $36 \pm 4^{\circ}[n=$ 50]; left $29 \pm 3.1^{\circ}[n=51]$ ). All deviations differed highly significantly from the $0^{\circ}$ plane $(p<000.1$ in all cases, Wilcoxon signed-rank test). No significant differences in rotation angles between the right and left hemispheres could be observed ( $p=0.28$ for CCT and rotation fluoroscopy, mixed-effects analysis with the Holm-Sidak multiple-comparisons test).

\section{Lead Rotation at Different Time Points}

We observed no significant changes in rotation angles between the first and second postoperative CCT (Fig. 3b; right, $p=0.45[n=12]$; left, $p=0.83[n=11]$; mixed-effects analysis with the Holm-Sidak multiple-comparisons test). 


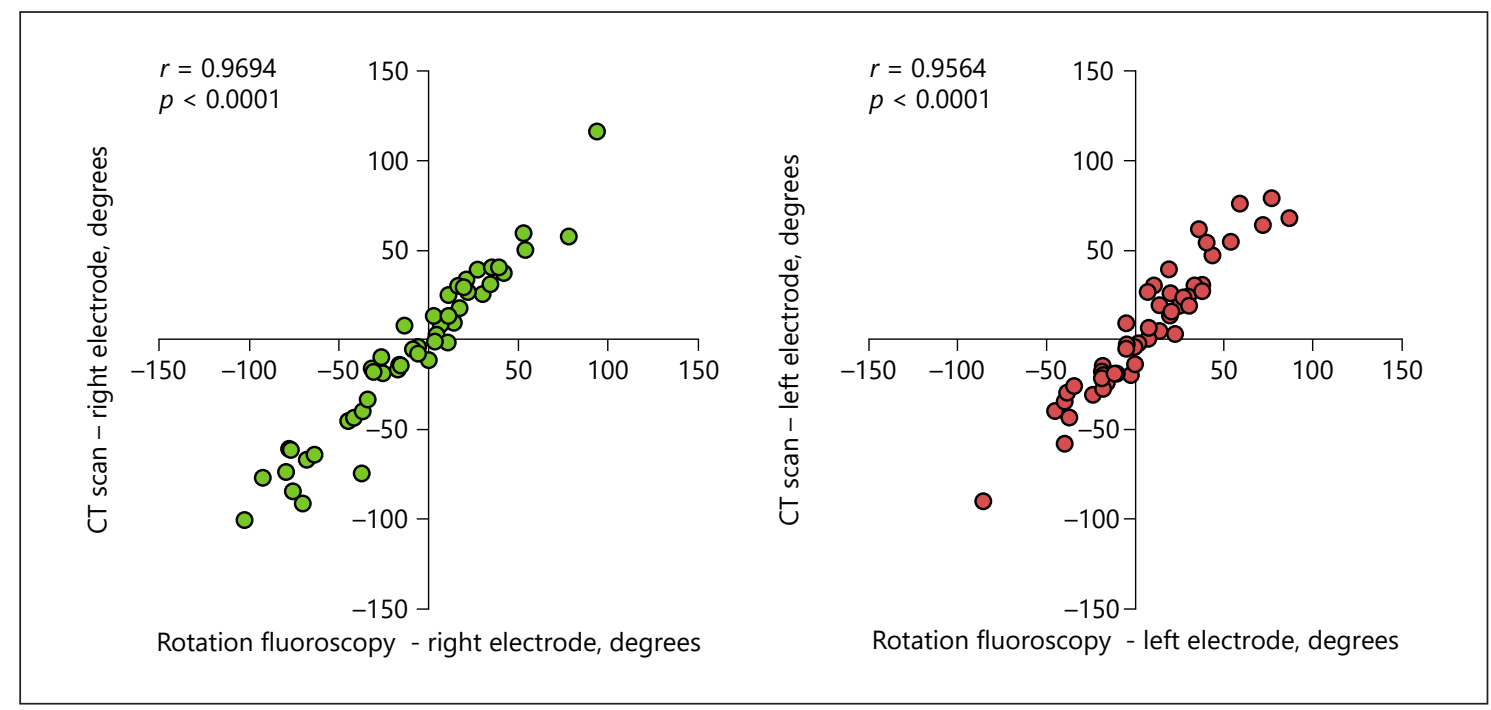

Fig. 5. Correlation curves of rotation angles determined by CCT (a) and rotation fluoroscopy (b). A highly significant correlation of measured values in patients who received both imaging modalities was observed (right: $p<0.0001, R^{2}=0.94, n=49$; left: $p<0.0001, R^{2}=0.91, n=51$; Pearson correlation coefficient with a two-sided $p$ value)

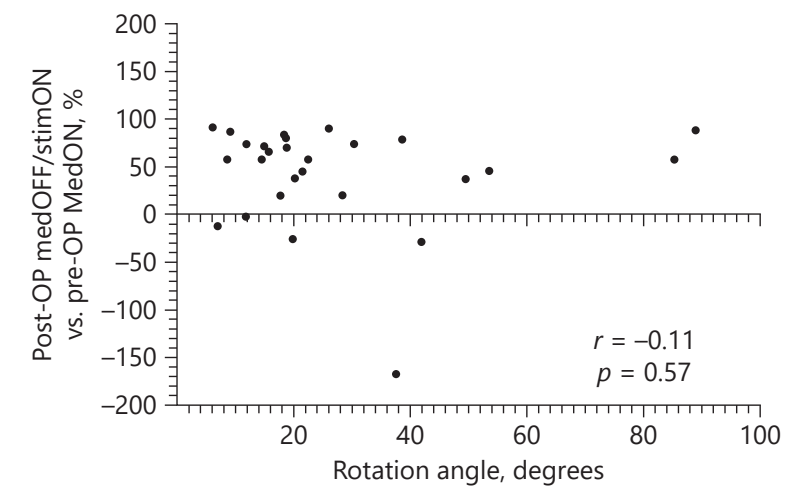

Fig. 6. Clinical impact of lead rotation angles. The lateralized motor outcome in PD patients vs. the rotation angle is shown. No significant correlation between lead rotation angles and contralateral clinical improvement by DBS (preoperative MedOFF vs. 1-year postoperative MedOFF/StimOFF MDS-UPDRSIII) could be observed ( $r=-0.1087, p=0.57, n=29$; nonparametric Spearman correlation).

The early 1 versus early 2 group (Fig. $3 \mathrm{~b}$; right, $p=0.6$ $[n=8] ;$ left, $p=0.9[n=7]$; mixed-effects analysis with the Holm-Sidak multiple-comparisons test) and the late 1 versus late 2 fluoroscopy group (Fig. 3 c; right, $p=0.98$
[ $n=7]$; left, $p=0.21[n=8]$; mixed-effects analysis with the Holm-Sidak multiple-comparisons test) exhibited no significant changes in lead rotation between the individual time points.

\section{Correlation of Rotation Angles Determined by CCT or Rotation Fluoroscopy}

Both methods of determining rotation angles were based on different imaging modalities and taken at different time points. We therefore performed the individual analyses on rotation angles for both methods separately. Since our data implies consistency of rotation angles during the postoperative follow-up, we felt that the values of different imaging modalities taken at different time points in a single individual could be correlated. Indeed, rotation angles measured by CCT versus rotation fluoroscopy exhibited a highly significant correlation (Fig. 5; right, $p<$ 0.0001, $R^{2}=0.94[n=49]$; left, $p<0.0001, R^{2}=0.91[n=$ 51]; Pearson correlation coefficient with a two-sided $p$ value).

\section{Correlation of Motor Outcome with Rotation Angles}

The mean clinical improvement in lateralized MDSUPDRSIII scores 1 year postoperatively was $37 \pm 10 \%$. No significant correlation between clinical outcome and the rotation angles of the corresponding contralateral hemisphere could be observed (Fig. 6, $r=-0.1087, p=0.57$ ). 


\section{Discussion}

As demonstrated by Dembek et al. [8], chronically implanted directional DBS leads consistently deviate to a significant extent from their intended intraoperative orientation. Reinacher et al. [7] showed that, most likely due to the flexibility of the lead's material, manual rotation of the proximal parts of the leads (such as performed intraoperatively under lateral fluoroscopy) is not transmitted to the lead tip in a predictable manner [7]. Hence, determination of the final lead rotation requires appropriate postoperative imaging. Obviously, directional programming should only be initiated after the leads reach their final rotation angle to avoid loss of therapeutic efficacy or adverse effects. The timing of this process, which could be driven by lead fixation, spring forces of the lead, or lead movements due to brain pulsation, has so far been unclear.

Our analysis did replicate the findings of Dembek et al. [8] concerning the significant deviations in lead rotation from their intended orientation $\left(0^{\circ}\right.$ anterior/posterior), irrespectively of the radiological method used to determine the lead rotation. Routinely, a postoperative CT scan is performed at our center roughly $24 \mathrm{~h}$ after DBS implantation, to exclude peri- or postoperative complications such as stroke, hemorrhage, or edema. This scan is usually the first imaging done after intraoperative lateral fluoroscopy. The comparison between the first and any following CT scans showed no relevant differences in lead rotation, arguing against relevant further lead rotations after the first postoperative day. To strengthen our findings using a second imaging modality, we also included a comparison of lead rotations in patients with multiple rotation fluoroscopies. This method was introduced in our department after the first reports of Reinacher et al. [7], to document the rotation angle of all implanted directional DBS leads. Therefore, some patients received their first rotation fluoroscopies retrospectively, several months after the operation, while others were scanned prospectively for programming within the first week postoperatively. The less stringent timing allowed grouping of patients into 3 categories based on timing. The results were similar to those of the CT imaging modality, i.e., no significant lead rotation could be observed after the first image had been taken during any phase of the postoperative period. Instead, repeated intraoperative lateral fluoroscopy (with all of the limitations of a single planar imaging modality) indicated relevant rotation of the lead after fixation to the skull in some cases. Therefore, it is possible that either manual rotation of the lead to its anterior facing position and/or bending of the flex-

Rotation Angles of Directional Leads ible lead for final fixation to the skull introduces a torque that releases completely within the first $24 \mathrm{~h}$ after surgery.

The lack of further lead rotation after postoperative day 1 is important for the timing of programming, which has to rely on a firm spatial relationship between target anatomy and contact location, regardless of whether clinical response testing or image-guided approaches for definition of the stimulated brain volume are used.

Our study represents the first comparison of the 2 currently available methods for determining the rotation angles of directional DBS leads in clinical practice. The first method, established by Dembek et al. [8], employs CCT scans, which are ubiquitous in neurosurgical and neurological departments and can exploit the postoperative scans routinely performed in most institutions. Optimal visualization of the artifacts requires the leads to be strictly positioned rectangularly to the gantry plane. In our experience, this is usually easily possible if considered during the imaging process, but it can severely impair the retrospective analysis if leads have been mispositioned during the scan. However, the most important limitation of this method is its inherent inability to differentiate between deviations from the intended anterior orientation and a rotation of $180^{\circ}$ to the opposite direction. Thus, a rotation angle of $+110^{\circ}$ might be mistaken for one of $-70^{\circ}$. Although extreme values of up to $117^{\circ}$ could be observed, $94 \%$ of all values were below $90^{\circ}$ and justify the assumption that the fundamental orientation facing forward with respect to the directional marker was usually preserved. Rotational fluoroscopy has the advantage of visualizing the directional marker above the 4 contacts in addition to the spaces between the directional contacts (iron sights). The marker itself also proved too imprecise to determine the exact rotation angle; nevertheless, it gives a basic idea of whether the lead rotation is above or below $\pm 90^{\circ}$. The method is also less sensitive to leads positioned with a flat angle. However, rotational fluoroscopy requires an angiography suite, usually only present in dedicated interventional radiology or neuroradiology departments, and a second X-ray exposure. From the rotation fluoroscopy, one can calculate a flat detector CT (FD CT), which allows the laterality of the leads to be determined and fusion to preoperative MRI based on bony landmarks. While FD CT offers sufficient quality to rule out larger intracerebral bleedings [11], its imaging quality of brain parenchyma is inferior to that of standard CCT and therefore unlikely to entirely replace postoperative CCT imaging. Despite the methodological advantages and disadvantages of each image modality, the correlation of the CT- and fluoroscopy-based rotation angles in our study under "real-life" 
conditions was very high, and thus every center might choose their preferred method based on resources and patient management pathways.

We found no negative impact of lead rotation (irrespectively of its extent) on clinical outcomes in PD. This has not been demonstrated before but it does not come as a big surprise given the fact that directional leads have proven their clinical efficacy in PD [12]. Clinical approaches to programming like monopolar reviews [4] simply identify the most effective contacts and are therefore not depending on knowledge of rotation angles.

Due to the rather low numbers of dystonia and tremor cases in our analysis, we refrained from analyzing these diseases.

In summary, we were able to demonstrate that the orientation of directional leads after the first postoperative day was stable, with no further subsequent rotation. Therefore, the first programming sessions (either clinical or image guided) can be conducted any time after this early postoperative period.

\section{Acknowledgement}

We would like to thank Peter Reinacher and Till Dembek for their assistance in establishing rotation fluoroscopy and the DiODe algorithm in our institution. We would also like to thank Deborah Nock (Medical WriteAway, Norwich, UK) for language editing of the final version of this work.

\section{Statement of Ethics}

The ethics committee of the University of Würzburg approved the retrospective scientific utilization of the clinical data (ethical vote 2019070404 ).

\section{Conflict of Interest Statement}

The authors claim no conflict of interests.

\section{Funding Sources}

The Open Access Publication Fund of the University of Würzburg supported this publication.

\section{Author Contributions}

Conception: F.L., F.S., J.V., C.M., and P.C. Design: F.L., F.S., D.E., T.N., P.F., J.V., C.M., and P.C. Funding: F.S., J.V., C.M., and P.C. Data collection and processing: F.L., F.S., D.E., T.M., T.N., P.F., C.M., and P.C. Analysis and Interpretation: F.L., D.E., T.M., T.N., J.V., C.M., and P.C. Writing: F.L., D.E., P.F., and P.C. Critical review and final approval: F.L., F.S., D.E., T.M., T.N., P.F., J.V., C.M., and P.C.

\section{References}

1 Frequin HL, Bot M, Dilai J, Scholten MN, Postma M, Bour LJ, et al. Relative Contribution of Magnetic Resonance Imaging, Microelectrode Recordings, and Awake Test Stimulation in Final Lead Placement during Deep Brain Stimulation Surgery of the Subthalamic Nucleus in Parkinson's Disease. Stereotact Funct Neurosurg. 2020;98(2):118-28.

2 O'Gorman RL, Jarosz JM, Samuel M, Clough C, Selway RP, Ashkan K. CT/MR image fusion in the postoperative assessment of electrodes implanted for deep brain stimulation. Stereotact Funct Neurosurg. 2009;87(4):20510.

3 Volkmann J, Herzog J, Kopper F, Deuschl G. Introduction to the programming of deep brain stimulators. Mov Disord. 2002;17(S3 Suppl 3):S181-7.

4 Picillo M, Lozano AM, Kou N, Puppi Munhoz R, Fasano A. Programming Deep Brain Stimulation for Parkinson's Disease: The Toronto Western Hospital Algorithms. Brain Stimul. 2016 May-Jun;9(3):425-37.
5 Pavese N, Tai YF, Yousif N, Nandi D, Bain PG. Traditional trial and error versus neuroanatomic 3-dimensional image software-assisted deep brain stimulation programming in patients with Parkinson disease. World Neurosurg. 2020 Feb;134:e98-102.

6 Dembek TA, Reker P, Visser-Vandewalle V, Wirths J, Treuer H, Klehr M, et al. Directional DBS increases side-effect thresholds-A prospective, double-blind trial. Mov Disord. 2017 Oct;32(10):1380-8

7 Reinacher PC, Krüger MT, Coenen VA, Shah $M$, Roelz R, Jenkner C, et al. Determining the Orientation of Directional Deep Brain Stimulation Electrodes Using 3D Rotational Fluoroscopy. AJNR Am J Neuroradiol. 2017 Jun; 38(6):1111-6.

8 Dembek TA, Hoevels M, Hellerbach A, Horn A, Petry-Schmelzer JN, Borggrefe J, et al. Directional DBS leads show large deviations from their intended implantation orientation. Parkinsonism Relat Disord. 2019 Oct;67:11721.
9 Hellerbach A, Dembek TA, Hoevels M, Holz JA, Gierich A, Luyken K, et al. DiODe: Directional Orientation Detection of Segmented Deep Brain Stimulation Leads: A Sequential Algorithm Based on CT Imaging. Stereotact Funct Neurosurg. 2018;96(5):335-41.

10 Horn A, Kühn AA. Lead-DBS: a toolbox for deep brain stimulation electrode localizations and visualizations. Neuroimage. 2015 Feb; 107:127-35.

11 Leyhe JR, Tsogkas I, Hesse AC, Behme D, Schregel K, Papageorgiou I, et al. Latest generation of flat detector $\mathrm{CT}$ as a peri-interventional diagnostic tool: a comparative study with multidetector CT. J Neurointerv Surg. 2017 Dec;9(12):1253-7.

12 Vitek JL, Jain R, Chen L, Tröster AI, Schrock LE, House PA, et al. Subthalamic nucleus deep brain stimulation with a multiple independent constant current-controlled device in Parkinson's disease (INTREPID): a multicentre, double-blind, randomised, sham-controlled study. Lancet Neurol. 2020 Jun;19(6): 491-501. 